\title{
OSCILLATIONS OF DELAY DIFFERENTIAL EQUATIONS
}

\author{
K. GOPALSAMY ${ }^{1}$ and G. LADAS ${ }^{2}$
}

(Received 30 July 1989; revised 28 February 1990)

\begin{abstract}
Sufficient conditions are established for all solutions of the linear system

$$
\frac{d y_{i}(t)}{d t}+\sum_{j=1}^{n} q_{i j} y_{j}\left(t-\tau_{i j}\right)=0, \quad i=1,2, \ldots, n,
$$

to be oscillatory, where $q_{i j} \in(-\infty, \infty), \tau_{j j} \in(0, \infty), i, j=1,2, \ldots, n$.
\end{abstract}

\section{Introduction}

Consider the system of delay differential equations

$$
\frac{d y_{i}(t)}{d t}+\sum_{j=1}^{n} q_{i j} y_{j}\left(t-\tau_{i j}\right)=0, \quad i=1,2, \ldots, n
$$

where the coefficients are real numbers and the delays are positive real numbers. We say that a solution

$$
y(t)=\left[\begin{array}{c}
y_{1}(t) \\
y_{2}(t) \\
\vdots \\
y_{n}(t)
\end{array}\right]
$$

of (1) oscillates if for some $i \in(1,2, \ldots, n), y_{i}(t)$ has arbitrarily large zeros. A solution $y(t)$ of $(1)$ is said to be nonoscillatory if there exists a $t_{0} \geq 0$ such that for each $i=1,2, \ldots, n, y_{i}(t) \neq 0$ for $t \geq t_{0}$. The aim of this brief paper is to derive a set of sufficient conditions for all solutions

${ }^{1}$ School of Mathematics, Flinders University, Bedford Park, S. A. 5042.

${ }^{2}$ Department of Mathematics, University of Rhode Island, Kingston, R. I., U.S.A.

(C) Copyright Australian Mathematical Society 1991, Serial-fee code 0334-2700/91 
of (1) to oscillate. Our result is an extension of a result of Gopalsamy in [2], where only bounded solutions of systems like (1) have been considered. For references concerning the oscillation of systems, the reader is referred to the references in [2].

\section{Sufficient conditions for oscillation}

The following lemma will be useful in the proof of our theorem below.

Lemma 1. Assume that (1) has a nonoscillatory solution (2). Then there are numbers

$$
\delta_{i} \in\{-1,1\} \text { for } i=1,2, \ldots, n
$$

such that the system

$$
\frac{d z_{i}(t)}{d t}+\sum_{j=1}^{n} p_{i j} z_{j}\left(t-\tau_{j j}\right)=0
$$

where

$$
p_{i j}=\frac{\delta_{j}}{\delta_{i}} q_{i j} \quad \text { for } i, j=1,2, \ldots, n
$$

has a nonoscillatory solution $\left[z_{1}(t), z_{2}(t), \ldots, z_{n}(t)\right]^{\top}$ with eventually positive components $z_{i}(t), i=1,2, \ldots, n$.

Proof. The components $y(t)$ of (2) are positive or negative eventually. That is, there exists a $T \geq 0$ such that $y_{i}(t) \neq 0$ for $t \geq T$ and $i=1,2, \ldots, n$. Set $\delta_{i}=\operatorname{sign}\left[y_{i}(t)\right], \quad i=1,2, \ldots, n$ and $t \geq T$. It is now easy to see that

$$
z(t)=\left[\delta_{1} y_{1}(t), \delta_{2} y_{2}(t), \ldots, \delta_{n} y_{n}(t)\right]^{\top}
$$

satisfies (3) and $\delta_{i} y_{i}(t)>0$ for $i=1,2, \ldots, n$ and $t \geq T$.

The next result is concerned with the asymptotic behaviour of nonoscillatory solutions of (1).

LEMmA 2. Consider the system (1) and suppose that the constant coefficients of (1) satisfy

$$
q=\min _{1 \leq i \leq n}\left[q_{i i}-\sum_{\substack{j=1 \\ j \neq i}}^{n}\left|q_{j i}\right|\right]>0 .
$$

Then every nonoscillatory solution $y(t)=\left(y_{1}, y_{2}, \ldots, y_{n}\right)$ satisfies

$$
\lim _{t \rightarrow \infty} y_{i}(t)=0 \text {. }
$$


Proof. Clearly (6) is also satisfied with the $q_{i j}$ replaced by the respective $p_{i j}$ of (4). From this and (5) it suffices to prove the lemma for nonoscillatory solutions of (2) with eventually positive components. Let us assume that there is a $t_{0} \geq 0$ such that $y_{i}(t)>0$ for $t \geq t_{0}, i=1,2, \ldots, n$. If we let

$$
w(t)=\sum_{j=1}^{n} y_{j}(t), \quad t \geq t_{0}
$$

then

or

$$
\frac{d w(t)}{d t}+\sum_{i=1}^{n} \sum_{j=1}^{n} q_{i j} y_{j}\left(t-\tau_{j j}\right)=0
$$

$$
\begin{aligned}
\frac{d w(t)}{d t}+\sum_{i=1}^{n} q_{i i} y_{i}\left(t-\tau_{i i}\right) & =-\sum_{i=1}^{n} \sum_{\substack{j=1 \\
j \neq i}}^{n} q_{i j} y_{j}\left(t-\tau_{j j}\right) \\
& \leq \sum_{i=1}^{n} \sum_{\substack{j=1 \\
j \neq i}}^{n}\left|q_{j i}\right| y_{i}\left(t-\tau_{i i}\right) .
\end{aligned}
$$

It follows from (8) that

$$
\frac{d w(t)}{d t}+\sum_{i=1}^{n}\left\{q_{i i}-\sum_{\substack{j=1 \\ j \neq i}}^{n}\left|q_{j i}\right|\right\} y_{i}\left(t-\tau_{i i}\right) \leq 0 .
$$

An integration of both sides of (9) leads to

$$
w(t)+q \int_{t_{0}+\tau}^{t} \sum_{i=1}^{n} y_{i}\left(s-\tau_{i i}\right) d s \leq w\left(t_{0}+\tau\right)
$$

where $\tau=\max _{1 \leq j \leq n} \tau_{j j}$. A consequence of $(10)$ is that $w$ is bounded and $y_{i} \in L_{1}\left(t_{0}+\tau, \infty\right)$ for $i=1,2, \ldots, n$. From the boundedness of $w$ one can conclude that of $y_{i}$ since $w(t)=\sum_{i=1}^{n} y_{i}(t)$ and $y_{i}(t)>0$ eventually. It will now follow from (1) that $\dot{y}_{i}$ is bounded for $t \geq \tau$, and therefore $y_{i}$ is uniformly continuous on $[0, \infty)$. The uniform continuity of $y_{i}$ on $[0, \infty)$, the eventual positivity of $y_{i}$ and the integrability of $y_{i}$ on a halfline together with a lemma of Barbalat [1], will imply that $\lim _{t \rightarrow \infty} y_{i}(t)=$ $0, i=1,2, \ldots, n$ and this completes the proof.

ThEOREM. Let $q_{i j} \in(-\infty, \infty), \tau_{j j} \in(0, \infty), i, j=1,2, \ldots, n$. If

$$
q \tau_{*}>\frac{1}{e} \text { where } q=\min _{1 \leq i \leq n}\left(q_{i i} \sum_{\substack{j=1 \\ j \neq i}}^{n}\left|q_{j i}\right|\right), \tau_{*}=\min _{1 \leq i \leq n} \tau_{i i}
$$

then every solution of (1) oscillates. 
Proof. Assume for the sake of a contradiction that (1) has a nonoscillatory solution (2). In view of Lemma 1 we can assume that the components of $y_{i}(t)$ are eventually positive for $i=1,2, \ldots, n$. We have directly from (1) that

$$
\sum_{i=1}^{n} \frac{d y_{i}(t)}{d t}+\sum_{j=1}^{n} \sum_{i=1}^{n} q_{i j} y_{j}\left(t-\tau_{j j}\right)=0
$$

which satisfies

$$
\sum_{i=1}^{n}\left[\frac{d y_{i}(t)}{d t}\right]+\sum_{i=1}^{n}\left(q_{i i}-\sum_{\substack{j=1 \\ j \neq i}}^{n}\left|q_{j i}\right|\right) y_{i}\left(t-\tau_{i i}\right) \leq 0 .
$$

We have from (12) that $w(t)=\sum_{i=1}^{n} y_{i}(t)$ satisfies

$$
\frac{d w(t)}{d t}+\sum_{i=1}^{n}\left[q_{i i}-\sum_{\substack{j=1 \\ j \neq i}}\left|q_{j i}\right|\right] y_{i}\left(t-\tau_{i i}\right) \leq 0 .
$$

Integrating both sides of (13) over $(t, \infty)$ and using the fact

$$
w(t) \rightarrow 0 \quad \text { as } t \rightarrow \infty \quad\left(\text { since } y_{i}(t) \rightarrow 0, i=1,2, \ldots, n\right)
$$

we derive that

$$
-w(t)+q \int_{t}^{\infty} \sum_{i=1}^{n} y_{i}\left(s-\tau_{i i}\right) \leq 0
$$

and this leads to

$$
w(t) \geq q \int_{t}^{\infty} \sum_{i=1}^{n} y_{i}\left(s-\tau_{i i}\right) d s .
$$

It is found from (15) that

$$
w(t) \geq q \int_{t-\tau_{*}}^{\infty} \sum_{i=1}^{n} y_{i}(s) d s, \quad \tau_{*}=\min _{1 \leq i \leq n} \tau_{i i}
$$

or

$$
w(t) \geq q \int_{t-\tau}^{\infty} w(s) d s
$$

Now we let

$$
F(t)=\int_{t-\tau_{\star}}^{\infty} w(s) d s
$$


and derive from (17) and (18) that

$$
\begin{aligned}
\frac{d F(t)}{d t} & =-w\left(t-\tau_{*}\right) \\
& \leq-q F\left(t-\tau_{*}\right) ; \quad t>2 \tau_{*} .
\end{aligned}
$$

It follows from (19) that $F$ is an eventually positive solution of

$$
\frac{d F(t)}{d t}+q F\left(t-\tau_{*}\right) \leq 0 ; \quad t>2 \tau_{*} .
$$

But it is well known (from Ladas and Stavroulakis [3]) that when (11) holds, (20) cannot have an eventually positive solution and this contradiction completes the proof.

\section{References}

[1] I. Barbalat, "Systemes d'equations differentielles d'oscillations nonlineaires," Rev. Roumain. Math. Pures Appl. 4 (1959) 267-270.

[2] K. Gopalsamy, "Oscillatory properties of systems of first order delay differential inequalities," Pacific J. Math. 128 (1987) 299-305.

[3] G. Ladas and I. P. Stavroulakis, “On delay differential inequalities of first order," Funkcialaj Ekvacioj. 25 (1982) 105-113. 